\title{
Effect of Bis-3-Sulfopropyl-Disulfide on the Characteristics of Trivalent Chromium Electrodeposition
}

\author{
Sol-Ji Song ${ }^{1}$, Sang Jin Ko ${ }^{1}$, Jae-Ryung Lee ${ }^{2}$, Jung-Gu Kim ${ }^{1, *}$ \\ ${ }^{1}$ School of Advanced Materials Science and Engineering, Sungkyunkwan University, 300 Chunchun- \\ Dong, Jangan-Gu, Suwon 440-746, Republic of Korea \\ ${ }^{2}$ Research and Development Laboratory, POSCO, 6261, Donghaean-ro, Nam-gu, Pohang 37859, \\ Republic of Korea \\ *E-mail: kimjg@skku.edu
}

doi: $10.20964 / 2018.08 .64$

Received: 3 April 2018 / Accepted: 7 June 2018 / Published: 5 July 2018

\begin{abstract}
This study examined the effect of bis-3-sulfopropyl-disulfide (SPS) addition to chromium sulfate solution on the characteristics of trivalent chromium electrodeposition. To investigate the effect of SPS on current efficiency, electrodeposition was carried out on the Invar surface at a constant current over time. Electrochemical tests, surface and solution analyses, density functional theory calculation, and Vickers micro-hardness tests were conducted to identify the SPS mechanism and evaluate its properties. SPS addition improved the electrodeposition current efficiency more than two-fold by increasing the nucleation of the electrode and changing the Cr-complex in solution to increase the bonding length between trivalent chromium ions and water molecules. Also, the surface hardness and corrosion resistance of the electrodeposited specimen were improved in the SPS-containing solution.
\end{abstract}

Keywords: Trivalent chromium; Electrodeposition; Bis-3-sulfopropyl-disulfide; Current efficiency

\section{FULL TEXT}

(C) 2018 The Authors. Published by ESG (www.electrochemsci.org). This article is an open access article distributed under the terms and conditions of the Creative Commons Attribution license (http://creativecommons.org/licenses/by/4.0/). 